of polemical discord. Solid advances have been made in recent years, notably with the electron microscope; and it is interesting to find that the processes of transfer and synthesis of yolk components are so varied that virtually all the disputed claims of the past can now be justified. Three sections, development of apterygote insects by C. Jura, and of hemimetabolous and holometabolous insects by D. T. Anderson, contain representative descriptive accounts of all the classic embryology of insects. It is valuable to have all this material so brought together. The final chapter of Volume 1 deals with polyembryony in insects by O. M. Ivanova-Kasasa fascinating subject now fully reviewed for the first time.

The book as a whole is dedicated to Professor Friedrich Seidel who, with his numerous students, has been the chief architect of experimental embryology in insects. Volume 2 opens with a 150-page chapter in which S. J. Counce reviews in detail "the causal analysis of insect embryogenesis". It must be admitted that little has been added to the basic principles since Seidel's classic work in the 1920s and 1930s. It is still a matter of "centres" the physiological activities of which are very uncertain. The succeeding section by $\mathbf{P}$. A. Lawrence is less concerned with completeness than with ideas. It provides a stimulating discussion of gradients in relation to differentiation. C. M. Child battled for years for recognition of the importance of gradients-but without much success. Gradients of what? As the review by Lawrence makes clear, it is possible to define the laws of gradients without knowing the true nature of the phenomenon. To discuss gradients of an imaginary something is no more and no less an invocation of the occult than is the idea of gravity-which, after nearly four hundred years, is still a useful concept.

Following upon the striking successes of E. Hadorn's researches, the study of the imaginal disks of Drosophila is a popular theme. It is well reviewed here by W. J. Gehring and R. Nöthinger. W. W. Doane contributes 175 pages of text with nearly 1,400 references on the role of hormones in insect development. My first reaction was that everything had been swept in, grain and chaff together. But having read the whole section I have to admit that, although exhaustive and somewhat exhausting, it is informed throughout by critical judgment. The book ends with a chapter by the second editor $\mathrm{C}$. $\mathrm{H}$. Waddington on the morphogenesis of patterns in Drosophila. This makes interesting reading, for much is based Waddington's own early work and leads on to more recent developments from it.

Altogether this is a book that con- tains a great deal that is of value-but it does not have the unity of a work of art. V. B. WigglesWORTH

\section{Fruit Fly Populations}

Behavioural and Ecological Genetics: a Study in Drosophila. By P. A. Parsons. Pp. vii +223 . (Clarendon: Oxford; Oxford University: London, March 1973.) $£ 6.50$.

THIS volume comprises a survey of recent work, with various species of Drosophila, which has provided evidence of genetic differences in behaviour and reaction to environmental conditions. Both field and laboratory studies are covered. The behaviour section deals with such topics as the effects of polygenic variation on behaviour traits as indicated by diallele crosses or selection for changes in geotaxis or mating speed. The second part is concerned with the evidence for genetic variation within and between populations in reaction to different kinds of environmental stress, laboratory studies on competition and the effects of genetic heterogeneity of various kinds on population growth, inversion polymorphism, the characteristics of marginal and central populations and also the contribution of behavioural and ecological differences to genetic isolation. The last section, although referred to as a synthesis, continues the story in terms of species distribution, interspecific competition and a discussion of the "adaptedness" of populations.

The literature survey is quite com. plete up to mid-1971, so this book serves a useful purpose as a source of references and a guide to the wide variety of genetic problems in ecology and behaviour which can be profitably studied with the aid of appropriate species of Drosophila.

The treatment of the material suggests that summaries of papers have played a large part in the compilation. The author very properly stresses the difficulties in defining adequate measures of the fitness of individuals and the "adaptedness" of populations, but one could go further. Given the frequent underestimation of the ecological complexity of the laboratory Drosophila culture, the often limited generality of measures of competition, inter-genotype interaction and so-called facilitation, which are all readily influenced by the particular conditions of the laboratory test as well as the genotypes which happen to be available, together with the frequent absence of adequate tests of repeatability, it would seem reasonable to encourage a robust scepticism, especially with regard to extrapolation to the even less understood natural situations. One suspects that, whatever the fashionable model, there would be no shortage of data which appeared to support it. When the interpretation of many experiments in ecological genetics is debatable it is premature to expect any author to provide a confident synthesis. But in that case it is all the more necessary to strike a probing, analytical attitude, otherwise the inexperienced reader will certainly be tempted to overestimate the number of problems which have been satisfactorily disposed of. It is probably significant that the book begins and ends with a reference to the remarkable Drosophila fauna of Hawaii which is beginning to yield up some of its evolutionary secrets as a consequence of a comprehensive, comparative study of many aspects of ecology and behaviour in both laboratory and the field. Integrated studies of this kind are needed for other groups of species which occur nearer home.

\section{F. W. ROBERTSON}

\section{Neutron Scattering}

Chemical Applications of Thermal Neutron Scattering. Edited by B. T. M. Willis. Pp. xiv +312 . (Oxford University: London, March 1973.) £9.50.

Although the opportunities for carrying out experiments in neutron scattering are few, and likely to remain so because of the expense of intense sources of neutrons, it is evident from this book that the rewards for perseverance are inimense.

Dr Willis's book brings together twelve chapters written at an advanced level by individual experts. The first two consider basic theory and experimental technique and the succeeding ten provide a very up-to-date picture of what can be achieved by neutron beam studies in different branches of "chemistry". Among the topics are molecular spectroscopy, polymers, liquid, glasses and non-stoichiometric compounds. It might be argued that some of these chemical applications have a decidedly physical flavour, but it is clearly evident that neutron scattering, like other good physical techniques, has now reached the stage at which it is in essential use by chemists. In view of the fundamental possibilities which the neutron provides this use is already overdue and it is often forgotten that, as a diffraction technique, neutrons are now half as old as $X$ rays.

The book arises from a series of lectures given at a Harwell Summer School and the editor is to be congratulated on having welded together his contributions with a coherence which is unusual in this kind of compilation. The symbolism and mode of theoretical approach which is set by the basic introductory chapter is faithfully followed by the later contributions. This greatly increases the value of the book to the non-expert and will permit him to learn a good deal 